\title{
Chapter 10 \\ Resilient Characteristics as Described in Empirical Studies on Health Care
}

\author{
Siv Hilde Berg and Karina Aase
}

\begin{abstract}
The concept of resilience needs greater empirical clarity. The literature on resilience in health care, published between 2006 and 2016, was reviewed with the aim of describing resilient characteristics in empirical studies. The chapter documents resilient characteristics at the individual, team, management, and organizational level. The characteristics were related to four overall conceptual categories: anticipation, sensemaking, trade-offs and adaptation. Based on empirical accounts resilience is described as a set of cognitive and behavioral strategies of individuals who enact resilience within an organizational context. The characteristics represented should be seen as examples of how resilience is described in the applied health care research, thus informing possible operationalization of resilience.
\end{abstract}

Keywords Anticipation $\cdot$ Sensemaking $\cdot$ Trade-off $\cdot$ Adaptation

Resilience in health care

\subsection{Background}

Health care has become a major field of focus for resilience studies accounting for a considerable amount of the empirical literature. However, a common model for operationalization has not been used in the applied research. This may relate to the lack of conceptual clarity. Several diverse definitions of resilience have been proposed

\footnotetext{
S. H. Berg (凶)

Division of Adult Mental Health, Sandnes DPS, Stavanger University Hospital, Postveien 181, N-4307 Stavanger, Norway

e-mail: siv.h.berg@uis.no

K. Aase

Centre for Resilience in Healthcare, Faculty of Health Sciences,

University of Stavanger, P.O. Box. 8600 Forus, N-4036 Stavanger, Norway

e-mail: karina.aase@uis.no 
over the last decade (e.g. [1, 2]), and researchers argue whether resilience is a unified concept or a compilation of multiple issues [3]. Concept formation is a prerequisite for any attempt to operationalize [4]. Operationalization entails a move from the abstract level to the empirical level, at which the ultimate goal is to find measures that validly and reliably capture the concept under study. The applied use of resilience reflects such action, and synthesizing this knowledge furthers the progress towards conceptualization and operationalization. This chapter therefore aims to synthesize resilient characteristics as described in empirical studies in health care.

We base our chapter on a content analysis of 15 empirical studies of resilience in health care. The literature searches were conducted as part of a larger study (see also [5]). The studies included were peer-reviewed articles or book chapters dated from January 2006 to February 2016. The studies drew from qualitative data within diverse clinical health care settings.

A directed content analysis was conducted [6]. The contents (e.g. resilient actions, attributes, abilities, contingencies, outcomes) of the included studies were collected according to predefined codes at different system levels (individual practitioners, health care teams, management, and organization). Inductive category development was conducted first within each system level. The categories representing resilient characteristics were held at the lowest possible abstraction level, keeping the concepts semantically close to the original findings where possible. Second, category development were conducted across levels to express conceptual categories at a higher abstraction level [7].

\subsection{Resilient Characteristics}

Resilient characteristics in the 15 included studies were categorized at individual, team, management and organizational level.

\subsubsection{Individual Practitioners}

Resilient characteristics at the individual level were anticipation, adaptation, sensemaking, and cognitive trade-offs.

Anticipation was described as an individual ability for health care professionals to enact resilience. Health care professionals anticipated gaps [8], work demands [9], and traits [10] in the clinical environments and handled each situation before it affected the patient. Pharmacists anticipated intervals of heavier demands and moved some of their work in order to reestablish a margin or buffer of safety to deal with urgent requests [9]. Preconditions for anticipation were related to both individual and situational demands. Ekstedt and Ödegård [8] found that health care professionals anticipated gaps by being sensitive to cues of fragility in the system. Cuvelier and Falzon [10] found that the level of physicians' experience and level of uncertainty 
affected their ability to anticipate an event in a paediatric anaesthesiology service. When the event overrode the physicians' ability to anticipate the situation, this was either related to physicians' inexperience or experienced physicians facing unknown novel situations.

Adaptations in clinical practice were understood as the result of coping with complexity in terms of unexpected situations, demands, variability attributed to the patient, or new technology $[8,11,12]$. Adaptations were described as an integrative part of daily practice to ensure good outcomes [8, 13]. Brattheim, Faxvaag, and Seim [14] described how variations were anticipated and planned for in vascular surgery. A similar connection was made by Cuvelier and Falzon [10], who found that the anesthesiologists had an anticipatory capacity that enabled them to define an envelope of potential variability before each operation. These findings connect the ability to anticipate and enact adaptations. In everyday clinical practice, the adaptations performed by practitioners were developing rules [11], adapting procedures [15], adding extra consultations and tests [14], conducting a "secret second handover" [16], or taking shortcuts and improvising [8]. Adaptations are performed differently according to the professional's level of competency, roles, and autonomy. Ross et al. [17] found that ward staff provided good outcomes by following treatment protocols to cope with narrow-focused tasks, while specialists could decide to go outside the protocol and take a holistic perspective with higher-level decisions, taking the complexity of each case into account.

Cuvelier and Falzon [10] studied resilient decisions in expected and unexpected events, where anesthesiologists had to adjust to unforeseen variability that required decision making under time constraints and with a high level of uncertainty. Their strategies depended on whether the situation was understood or not. In situations perceived as unexpected, a sense of what was happening was lost (i.e. "collapse of sensemaking" [18]). These findings demonstrate the act of sensemaking when facing unexpected events. Without identification of the problem at hand, a protocol could not directly deal with the event, and the anesthesiologists had to make cognitive tradeoffs, choosing between establishing a correct diagnosis before acting or choosing one of the possible protocols. When the individual understands that an event is changing from normal towards abnormal [19], or towards a crisis [12], adjustments can be made to act proactively to prevent the adverse event or crisis.

\subsubsection{Health Care Teams}

Resilient characteristics at the team level were categorized as managing trade-offs between competing goals, collaboration across specialists and collective sensemaking.

The ability to manage trade-offs between competing goals has been described as a dynamic decision-making process between professionals which collaborates in clinical care. Competing goals and tensions emerge between professionals with different roles, clinical aims, and goals [16, 17]. Tensions are described between the need for 
safety of the individual patient versus the safety of the patient in the community [16] or between patients' versus nurses' need for safety [15]. Further tensions emerge between professionals due to different goals in care [16]. In order to adapt to these tensions, health care teams make trade-offs based on their experience and decide what to sacrifice $[12,16]$. This decision-making process is described as flexible, dynamic (constantly under re-evaluation), and highly context dependent [15, 16]. Teamwork was considered as an adaptive response to ensure safe work to task demands that could not be met alone. Nurses considered that teamwork maximized their physical, cognitive and emotional resources to successfully manage work demands [15]. Paries et al. [12] introduced the concept of "coopetition", a merging of cooperation and competition, and proposed coopetition as a team resilient characteristic in an ICU understood as an ability to manage competing goals. The doctor can decide that a protocol needs to be adapted, but not be in charge of the implementation of the decision, and depend on the nurses performing the duties. Nurses can meet this request with resistance, as they are put in a risky situation by performing outside the protocol. Coopetition was the resilient response to the diversity of interests that emerged between different professionals.

Collaboration across specialists has been described as a resilient characteristic among specialists across disciplines related to anticipation, mitigation, and decision making. Collaborative cross checks comprise a strategy to monitor decision-making to detect erroneous assumptions and actions and prevent errors from happening. At least two people assess the accuracy and validity of others' assumptions and/or actions. The patterns observed in three health care incidents established that collaborative cross checks enhanced system resilience when the incoming fellow had specialized and interdisciplinary knowledge. The cross checks made the process more observable and explicit [20]. Collaboration across specialists was also found to be a resilient characteristic in diabetes care, in which the specialist teams detected problems early and reduced future risks [17]. These findings connect team expertise to the ability to anticipate and act reactively to problems.

Strategies and activities to achieve collective sensemaking have been described as resilient characteristics within and between health care teams. The medical visit is an example of a daily activity, which is important for sensemaking within the intensive care team. A collective understanding of the perceived clinical behavior of the patient was built during the medical visit. The shared understanding obtained from a diversity of professionals observations improved the anticipation of future actions to take when faced with clinical complexity [12]. Direct means of communication such as contacting a clinic directly was described as a strategy to obtain a shared common ground between clinics in the diagnostic process of lung and colorectal cancer [21]. Verbal communication was also preferred to ensure shared understanding during handovers in emergency care. Clinicians felt they could not simply rely on documentation. However, in the dialogue health care professionals could highlight important information, discuss their concerns and question information [16]. 


\subsubsection{Management}

A few studies described resilient characteristics at the management level, categorized as anticipatory regulation, and crisis management adjustments.

A resilient characteristic found in the ICU was the anticipatory regulation performed by the managing assistant nurse who anticipated patient flow and work demands in the ICU and prevented capacity crisis [12]. Miller et al. [22] found that each level of management aimed to provide staffing resources appropriate to the anticipated patient demand. A strategy to avoid decompensation in periods with increased patient demands beyond the anticipated levels was to maintain and develop compensatory buffers consisting of staff that could be called upon in periods of high demand.

Facing unexpected events or a crisis, crisis management adjustments from normal situations towards crisis management involve acknowledging the need to shift from one mode to the other. The crisis response depended on whether the ICU was facing a capacity crisis or a complexity crisis. Crisis management in a capacity crisis was characterized by delegation and decentralization relying on the competence and the sensemaking skills of the teams; however, crisis management in a complexity crisis was characterized by mobilization to increase the level of expertise [12]. These findings relate to sensemaking in cases of unexpected events.

\subsubsection{Organization}

Resilience characteristics at the organizational level were mainly reflected in the discussion part of the extant studies. Resilient characteristics were related to organizational outcomes of resilience and organizational conditions that supported resilient performance.

Although good organizational outcomes of resilient performance were described at the department level, this was not always the case at the organizational level when considering the system as a whole beyond the individual components of the organization. The adaptations that are made can make sense locally, but the outcomes are not necessarily successful at a higher level. Resilient performance can ironically lead to brittleness at the organizational level [11, 16, 21]. Laugaland and Aase [23] found that the outcomes of the adjustments imposed by a system reform were perceived as successful from the hospital's perspective, with mixed outcomes from the primary care perspective, and with poor outcomes from the patient's perspective.

Another characteristic described is the vulnerability emerging when the organization relies heavily on resilient expertise and adaptations at the sharp end, leaving the system brittle [8, 9, 17]. By relying on the specialists' expertise, Ross et al. [17] found that the system was threatened by skill erosion among the ward staff. Brattheim et al. [14] and Ekstedt and Ödegard [8] found that resilient performance at the individual level compensated for the lack of resilience in the health care organi- 
zation and then became "invisible" to managers. Patterson and Wears [9] studied hospital pharmacies, in which the adaptive capacity was exhausted. They found that the system had relied on individuals working at their maximum capacity every day, thereby stretching the system into brittleness and patterns of decompensation. Without the adaptive capacity, the system was not able to respond to unusual demands or a crisis.

Suggestions of how organizational conditions (e.g. structural and cultural) can enhance resilience have been presented in the literature. To enhance individuals' capability for awareness in vascular surgery, IT-based process support can be designed to give real-time process information concerning the actual execution and status of the ongoing clinical process [14]. Smith et al. [21] emphasized the importance of supporting the blunt-end administration's awareness of demands and challenges at the sharp end of the system to better anticipate and adapt to problems.

\subsubsection{Conceptualization of Resilience in Health Care}

The characteristics described in the empirical studies of resilience are interconnected both within and across levels, according to four overall conceptual categories: anticipation, sensemaking, trade-offs, and adaptations. These four conceptual categories are cognitive and behavioral strategies of individuals who enact resilience within an organizational context. The strategies can be studied at individual, team and management level.

Anticipation is an act of looking forward and relates to the future, which enables individuals to enact proactively and prevent adverse events from happening. At the individual level, practitioners anticipate threats, and at the team level, collaboration among specialists implies anticipation. At the management level, anticipation of demands on the system and regulation of these demands to prevent crisis is included.

Sensemaking is the perception of something that is experienced with regard to the current situation. A sense of what is happening is needed to adapt and make trade' offs in both normal practice and in unexpected events. Individual practitioners make sense of unexpected events, team members share information to understand complex cases and managers make sense of unexpected events and crisis to conduct the adjustments needed.

Trade-offs relate to the act of decision-making and is an adaptive response toward the inherent complexity in every day practice. Two types of trade-offs are common: cognitive trade-offs at the individual level and trade-offs between competing goals at the team level.

Adaptations are adjustments made in work practices as a result of coping with complexity. Diverse adaptations are common at the individual level such as improvisations and adjustment of procedures. At the team level, adaptations are made to manage tensions between goals, and at the management level, adaptations concern the shift from normal mode to crisis management. 
Resilience are enacted within an organizational context, thus individual strategies are influenced by multiple organizational conditions at different system levels. Further, organizational outcomes of enacted resilience are evident locally, across department and institutional levels. The perceptions of such outcomes varies depending on the stakeholder and system level.

\subsection{Conclusion}

Our conceptualization of resilience in health care based on existing empirical accounts represents resilience as a set of cognitive and behavioral strategies of individuals who enact resilience within an organizational context. The conceptualisation adds conceptual clarity in terms of the applied uses of resilience in health care, thus informing possible operationalization of resilience.

Although the setting in this chapter is limited to health care, theoretical generalizations can be made to other industries and settings. Our analysis finds common characteristics across the system levels, i.e. for individual practitioners, within health care teams, and at the management level. These resilient characteristics are expressed as anticipation, sensemaking, trade-offs, and adaptations. Our findings suggest that, although resilience can be considered as a diverse and interconnected concept, it is not necessarily differently expressed at different levels.

Despite the existence of several frameworks for resilience, which represent concepts for resilience at the organizational level, (i.e. "the four cornerstones of resilience" [2]), the included studies did not document any resilient characteristics at this level. Operationalizing and empirically studying resilience at this system level appear to present challenging topics within the research field. As such, the challenge is how to operationalize and study resilience empirically as a system characteristic, not merely expressed as a sum of individuals who enact resilience.

The outcomes described at the organizational level demonstrate that resilience is not necessarily positive for safety considering the system as a whole. This implies the need to address resilience across institutional borders. To better understand the nuances of resilient characteristics more research is needed on how resilience is expressed within and across different system levels, as well as the outcomes of enacted resilience, and the organizational conditions to enhance resilience at multiple system levels.

\section{References}

1. A.W. Righi, T.A. Saurin, P. Wachs, A systematic literature review of resilience engineering: research areas and a research agenda proposal. Reliab. Eng. Sys. Saf. 141, 142-152 (2015)

2. E. Hollnagel, J. Braithwaite, R.L. Wears, Epilogue: how to make health care resilient, in Resilient Health Care, ed. by E. Hollnagel, J. Braithwaite, R.L. Wears (Ashgate, Farnham, 2013) 
3. D.D. Woods, Four concepts for resilience and the implications for the future of resilience engineering. Reliab. Eng. Sys. Saf. 141, 5-9 (2015)

4. G. Sartori, Concept misformation in comparative politics, in Concepts and Method in Social Science: The Tradition of Giovanni Sartori, ed. by D. Collier, J. Gerring (Routledge, New York, 2008)

5. S.H. Berg, K. Akerjordet, M. Ekstedt, K. Aase, Methodological strategies in resilient health care studies: an integrative review. Safety Sci 110, 300-312 (2018)

6. H.-F. Hsieh, S.E. Shannon, Three approaches to qualitative content analysis. Qual. Health Res. 15(9), 1277-1288 (2005)

7. P. Mayring, Qualitative content analysis: theoretical foundation, basic procedures and software solution (2014)

8. M. Ekstedt, S. Ödegård, Exploring gaps in cancer care using a systems safety perspective. Cogn. Technol. Work 17(1), 5-13 (2015)

9. M.D. Patterson, R.L. Wears, Resilience and precarious success. Reliab. Eng. Sys. Saf. 141, 45-53 (2015)

10. L. Cuvelier, P. Falzon, Coping with uncertainty: resilient decisions in anaesthesia, in Resilience Engineering in Practice: A Guidebook, ed. by E. Hollnagel, J. Pariès, D.D. Woods, J. Wreathall (Ashgate, Farnham, 2011)

11. C.P. Nemeth, M. Nunnally, M.F. O’Connor, M. Brandwijk, J. Kowalsky, R.I. Cook, Regularly irregular: how groups reconcile cross-cutting agendas and demand in healthcare. Cogn. Technol. Work 9(3), 139-148 (2007)

12. J. Paries, Resilience in intensive care units: the HUG case in resilient health care, in Resilient Health Care, ed. by E. Hollnagel, J. Braithwaite, R.L. Wears (Ashgate, Farnham, 2013)

13. A.S. Nyssen, A. Blavier, Investigating expertise, flexibility, and resilience in socio-technical environments: a case study in robotic surgery, in Resilient Health Care, ed. by E. Hollnagel, J. Braithwaite, R.L. Wears (Ashgate, Farnham, 2013)

14. B. Brattheim, A. Faxvaag, A. Seim, Process support for risk mitigation: a case study of variability and resilience in vascular surgery. BMJ Qual. Saf. 20(8), 672-679 (2011)

15. V.J. O'Keeffe, M.R. Tuckey, A. Naweed, Whose safety? Flexible risk assessment boundaries balance nurse safety with patient care. Saf. Sci. 76, 111-120 (2015)

16. M. Sujan, P. Spurgeon, M. Cooke, The role of dynamic trade-offs in creating safety - a qualitative study of handover across care boundaries in emergency care. Reliab. Eng. Sys. Saf. 141, 54-62 (2015)

17. A.J. Ross, J.E. Anderson, N. Kodate, K. Thompson, A. Cox, R. Malik, Inpatient diabetes care: complexity, resilience and quality of care. Cogn. Technol. Work 16(1), 91-102 (2014)

18. K.E. Weick, Collapse of sensemaking in organizations: the Mann Gulch disaster. Adm. Sci. Quaterly 38(4), 628-652 (1993)

19. S.W. Dekker, J. Bergström, I. Amer-Wåhlin, P. Cilliers, Complicated, complex, and compliant: best practice in obstetrics. Cogn. Technol. Work 15(2), 189-195 (2013)

20. E.S. Patterson, D.D. Woods, R.I. Cook, M.L. Render, Collaborative cross-checking to enhance resilience. Cogn. Technol. Work 9(3), 155-162 (2007)

21. M.W. Smith, T.D. Giardina, D.R. Murphy, A. Laxmisan, H. Singh, Resilient actions in the diagnostic process and system performance. BMJ Qual. Saf. 22(12), 1006-1013 (2013)

22. A. Miller, Y. Xiao, Multi-level strategies to achieve resilience for an organisation operating at capacity: a case study at a trauma centre. Cogn. Technol. Work 9(2), 51-66 (2007)

23. K. Laugaland, K. Aase, The demands imposed by a health care reform on clinical work in transitional care of the elderly: a multi-faceted Janus, in Resilient Health Care Volume 2: The Resilience of Everyday Clinical Work, ed. by R.L. Wears, E. Hollnagel, J. Braithwaite (Ashgate, Farnham, 2015) 
Open Access This chapter is licensed under the terms of the Creative Commons Attribution 4.0 International License (http://creativecommons.org/licenses/by/4.0/), which permits use, sharing, adaptation, distribution and reproduction in any medium or format, as long as you give appropriate credit to the original author(s) and the source, provide a link to the Creative Commons license and indicate if changes were made.

The images or other third party material in this chapter are included in the chapter's Creative Commons license, unless indicated otherwise in a credit line to the material. If material is not included in the chapter's Creative Commons license and your intended use is not permitted by statutory regulation or exceeds the permitted use, you will need to obtain permission directly from the copyright holder.

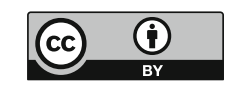

